\title{
Preparation of Erythromycin Analogs Having Functional Groups at C-15
}

\author{
Gary W. Ashley, Mark Burlingame, Ruchir Desai, Hong Fu, Tim Leaf, Peter J. Licari, \\ Chau Tran, Darren Abbanat, Karen Bush, Mark Macielag
}

Received: April 6, 2006 / Accepted: July 6, 2006

(C) Japan Antibiotics Research Association

\begin{abstract}
Chemobiosynthesis has been used to prepare analogs of erythromycins having unique functional groups at the 15-position. Using diketide thioester feeding to genetically engineered Streptomyces coelicolor, analogs of 6-deoxyerythronolide B were prepared having 15-fluoro, 15-chloro, and 15-azido groups. Bioconversion using a genetically engineered mutant of Saccharopolyspora erythraea was used to produce 15-fluoroerythromycin A and 15-azidoerythromycin A. These new erythromycin analogs provide antibacterial macrolides with unique physicochemical properties and functional groups that allow for selective derivatization.
\end{abstract}

Keywords erythromycin, fluorine, azide, antibacterial, genetic engineering, polyketide

\section{Introduction}

The search for new macrolide antibacterial agents has been driven by the need to improve the spectrum, stability, and physicochemical properties of existing antibiotics. Erythromycin A (1) has been successfully used to treat respiratory infections for over 50 years, but suffers from marked instability, poor bioavailability, undesired gastrointestinal side effects, and an increasingly limited spectrum of activity with the rise in macrolidelincosamide-streptogramin B MLS(B)-resistant bacteria. Chemical derivatization of erythromycin $\mathrm{A}$ has now been thoroughly investigated as a means of overcoming most of these limitations. For example, 6- $O$-methylation to produce clarithromycin and ring expansion of the 9-oxime to produce azithromycin have been highly successful at stabilizing the macrolide, but with no marked improvements in spectrum. Chemical conversion of $\mathbf{1}$ to the ketolides telithromycin and cethromycin has improved both the stability and spectrum of activity, although at the expense of rather complex chemistry. Replacement of the cladinose sugar with various acyl groups has yielded acylides with improved spectrum, in part due to overcoming inducible MLS(B) resistance [1]. Given the extensive medicinal chemistry that has been performed, there appear to be few remaining positions on the erythromycin scaffold that have not been modified. Further development of erythromycin thus requires radically new technology.

The production of novel erythromycins through chemobiosynthesis has been previously reported $[2,3]$. In this process, synthetic starter units are introduced into the erythromycin polyketide by feeding a diketide analog to an engineered strain in which the ketosynthase domain of the initial polyketide synthase module has been inactivated. This technology has been used to make chemically rather conservative changes to the erythromycin structure, for example replacing the starting propionyl group with a butyryl group. Another technology using a polyketide synthase (PKS) having a genetically engineered load domain from the avermectin PKS has also provided erythromycins with chemically conservative changes to the starter unit, such as replacing the starting propionyl group with a cyclopentyl group [4]. These erythromycins
G. W. Ashley (Corresponding author), M. Burlingame, R. Desai, H. Fu, T. Leaf, P. J. Licari, C. Tran: Kosan Biosciences, Inc., 3832, Bay Center Place, Hayward, California, 94545 USA, E-mail: Ashley@kosan.com
D. Abbanat, K. Bush, M. Macielag: Johnson \& Johnson Pharmaceutical Research and Development, L.L.C., Raritan, New Jersey, 08869 USA 


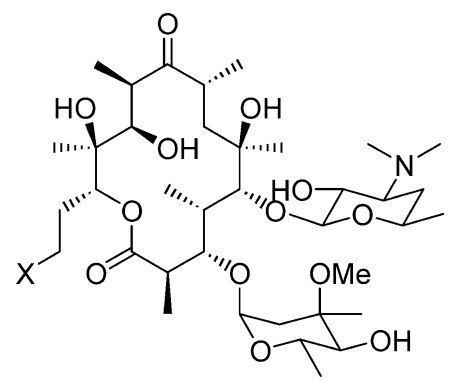

(1) $X=H$

(2) $X=F$

(3) $X=N_{3}$

Fig. 1 Erythromycin A and C-15 substituted erythromycins.

have generally shown similar to reduced activity relative to erythromycin A. The C-15 hydroxylation of the erythromycin derivative 6-O-methylerythromycin has also been reported, however 15-hydroxyerythromycin itself appears to be unreported $[5,6]$.

We recognized that it might be possible to use chemobiosynthesis technology to introduce functional groups that will provide erythromycin analogs having significantly altered physicochemical properties or having unique chemical handles that allow for selective derivatization. We describe here the preparation and properties of two such analogs, 15-fluoroerythromycin A (2) and 15-azidoerythromycin A (3).

\section{Materials and Methods}

\section{General}

NMR spectra were obtained in $\mathrm{CDCl}_{3}$ at $298 \mathrm{~K}$ using a Bruker DMX-400 spectrometer fitted with a 3-mm proton-carbon probe (Nalorac). Exact masses were obtained using a Mariner time-of-flight mass spectrometer (Applied Biosystems) with electrospray ionization. Samples were introduced by flow injection using $1: 1$ $\mathrm{MeOH} / \mathrm{H}_{2} \mathrm{O}$ containing $5 \mu \mathrm{M} \mathrm{NH} \mathrm{N}_{4} \mathrm{OAc}$ and three standard compounds whose $\mathrm{m} / \mathrm{z}$ ratios were used to calibrate the exact mass. Optical rotations were measured using a Perkin-Elmer model 341 polarimeter with a $100-\mathrm{mm}$ pathlength cell. IR spectra were obtained from thin films prepared from evaporation of a $\mathrm{CH}_{2} \mathrm{Cl}_{2}$ solution using attenuated total reflection on a Perkin-Elmer Spectrum One FT-IR spectrophotometer. Erythromycin A for antibacterial testing was obtained from Sigma. Bacterial strains were from the American Type Culture Collection (ATCC) as indicated in Table 1, or were clinical isolates. MIC values for reference and test compounds were determined in
Mueller-Hinton broth (BBL) by the broth microdilution method according to NCCLS guidelines [7]. All fermentation medium components were from SigmaAldrich, with the exception of soya meal flour, which was obtained from Giusto's Specialty Foods, South San Francisco, CA, USA.

\section{Partition Coefficients}

$\log \mathrm{D}$ values were estimated by two methods. In the first, the individual compounds $\mathbf{1}$ and $\mathbf{2}$ were partitioned between 1-octanol and $0.1 \mathrm{M}$ sodium $N$-tris(hydroxymethyl)methyl2-aminoethanesulfonate (TES-Na ${ }^{+}$), $\mathrm{pH} 7.4$, and the amount of compound remaining in the aqueous phase was determined by HPLC analysis. In a second method, a mixture of equal amounts of $\mathbf{1}$ and $\mathbf{2}$ in $10 \mathrm{mM}$ sodium $N$-2-(hydroxyethyl)piperazine- $N^{\prime}$-2-ethanesulfonate (HEPES-Na ${ }^{+}$), pH 7.4, was stirred vigorously with an equal volume of buffersaturated 1-octanol for 24 hours, and the aqueous phase was analyzed by HPLC, revealing an $80: 20$ ratio of 2 to $\mathbf{1}$.

\section{3-Fluoropropanal (4)}

Trichloroisocyanuric acid $(17.0 \mathrm{~g}, 73 \mathrm{mmol})$ was added in $c a .1 \mathrm{~g}$ portions over 1 hour to a vigorously stirred mixture of 3-fluoropropanol $(15.6 \mathrm{~g}, 200 \mathrm{mmol}), \mathrm{NaHCO}_{3}(16.8 \mathrm{~g}$, $200 \mathrm{mmol})$, and TEMPO $(0.35 \mathrm{~g}, 2.2 \mathrm{mmol})$ in $\mathrm{CH}_{2} \mathrm{Cl}_{2}$ $(300 \mathrm{ml})$ and water $(10 \mathrm{ml})$. The temperature was kept at $20 \sim 25^{\circ} \mathrm{C}$ using a water bath. After addition was complete, the mixture was stirred until the orange color faded to a pale yellow (approximately 30 minutes). The resulting solution was decanted from the gummy white residue, filtered through a pad of silica gel $(5 \mathrm{~g})$, and dried over $\mathrm{MgSO}_{4}(10 \mathrm{~g})$ for 30 minutes to provide a solution of $\mathbf{4}$ in $\mathrm{CH}_{2} \mathrm{Cl}_{2} \cdot{ }^{1} \mathrm{H}$ NMR $\left(400 \mathrm{MHz}, \mathrm{CDCl}_{3}\right) \delta 9.82(1 \mathrm{H}$, br s, $\mathrm{CHO}), 4.78\left(2 \mathrm{H}, \mathrm{t}, J=46.4,5.6 \mathrm{~Hz}, \mathrm{CH}_{2} \mathrm{~F}\right), 2.84(2 \mathrm{H}, \mathrm{dt}$, $\left.J=25.6,5.6 \mathrm{~Hz}, \mathrm{CH}_{2} \mathrm{CO}\right)$.

The concentration of 4 was determined by dilution of $50 \mu \mathrm{l}$ of the solution into $300 \mu \mathrm{l}$ of $\mathrm{CDCl}_{3}$ and recording the ${ }^{1} \mathrm{H}$-NMR spectrum. The integral of the $\mathrm{CH}_{2} \mathrm{Cl}_{2}$ peak $(\delta$ 5.28) was taken as representing $31.2 \mathrm{M}$ protons in the neat solution, and compared to the integral of the aldehyde peak ( $\delta 9.82$ ) such that the concentration of $\mathbf{4}$ was estimated using the formula: [4]=(integral of $\mathrm{CHO}$ )/(integral of $\left.\mathrm{CH}_{2} \mathrm{Cl}_{2}\right) * 31.2$. This procedure provided an approximately $0.5 \mathrm{M}$ solution of 4 in $\mathrm{CH}_{2} \mathrm{Cl}_{2}$, which was stable for several weeks when stored at $-20^{\circ} \mathrm{C}$.

\section{3-Azidopropanal (5)}

Prepared according to [8]. ${ }^{1} \mathrm{H}$ NMR $\left(400 \mathrm{MHz}, \mathrm{CDCl}_{3}\right) \delta$ $9.81(1 \mathrm{H}, \mathrm{t}, J=1.0 \mathrm{~Hz}, \mathrm{CHO}), 3.62(2 \mathrm{H}, \mathrm{t}, J=6.3 \mathrm{~Hz}$, $\left.\mathrm{CH}_{2} \mathrm{~N}_{3}\right), 2.73\left(2 \mathrm{H}, \mathrm{dt}, J=1.0,6.5 \mathrm{~Hz}, \mathrm{CH}_{2} \mathrm{CO}\right) .{ }^{13} \mathrm{C} \mathrm{NMR}$ $\left(100 \mathrm{MHz}, \mathrm{CDCl}_{3}\right) \delta 199.2,44.5,42.8$. 
Table $\mathbf{1}$ In vitro activities of $\mathbf{1}, \mathbf{2}$, and $\mathbf{3}$ against selected pathogens

\begin{tabular}{|c|c|c|c|c|c|}
\hline & & \multicolumn{4}{|c|}{$\mathrm{MIC}(\mu \mathrm{g} / \mathrm{ml})$} \\
\hline & & $1^{a}$ & 2 & $1^{\mathrm{b}}$ & 3 \\
\hline \multicolumn{6}{|l|}{ S. pneumoniae } \\
\hline ATCC6301 & & 0.06 & 0.06 & 0.03 & 0.03 \\
\hline ATCC49619 & & 0.12 & 0.06 & 0.06 & 0.06 \\
\hline OC2540 & & $\leq 0.015$ & $\leq 0.015$ & $\leq 0.015$ & $\leq 0.015$ \\
\hline OC4445 & & 0.03 & 0.06 & 0.06 & 0.06 \\
\hline OC4821 & & 0.06 & 0.06 & 0.06 & 0.03 \\
\hline OC4869 & & 0.03 & 0.06 & 0.03 & 0.03 \\
\hline \multirow[t]{3}{*}{ OC4876 } & & 0.06 & 0.06 & 0.03 & 0.03 \\
\hline & Range & $\leq 0.015 \sim 0.12$ & $\leq 0.015 \sim 0.06$ & $\leq 0.015 \sim 0.06$ & $\leq 0.015 \sim 0.06$ \\
\hline & Median & 0.06 & 0.06 & 0.03 & 0.03 \\
\hline OC4438 & mef & 8 & 8 & 4 & 4 \\
\hline OC4439 & mef & 2 & 4 & 2 & 2 \\
\hline OC4429 & mef & 4 & 8 & 4 & 4 \\
\hline OC4436 & mef & 2 & 4 & 2 & 4 \\
\hline OC4421 & mef & 4 & 8 & 4 & 2 \\
\hline \multirow[t]{3}{*}{ OC4568 } & mef & 2 & 4 & 1 & 1 \\
\hline & Range & $1 \sim 8^{*}$ & $4 \sim 8$ & & $1 \sim 4$ \\
\hline & Median & $2 \sim 4$ & $4 \sim 8$ & $2 \sim 4$ & $2 \sim 4$ \\
\hline OC3604 & erm & $>16$ & $>16$ & $>16$ & $>16$ \\
\hline OC4051 & erm & $>16$ & $>16$ & $>16$ & $>16$ \\
\hline OC4432 & erm & $>16$ & $>16$ & $>16$ & $>16$ \\
\hline \multirow[t]{3}{*}{ OC4444 } & erm & 4 & 8 & 2 & 4 \\
\hline & Range & $2 \sim>16^{*}$ & $8 \sim>16$ & & $4 \sim>16$ \\
\hline & Median & $>16$ & $>16$ & $>16$ & $>16$ \\
\hline \multicolumn{6}{|l|}{ H. influenzae } \\
\hline ATCC49247 & & 2 & 2 & 1 & 0.5 \\
\hline ATCC49766 & & 2 & 2 & 1 & 0.5 \\
\hline OC4881 & & 8 & 8 & 4 & 2 \\
\hline OC4882 & & 8 & 8 & 8 & 2 \\
\hline OC4884 & & 4 & 4 & 4 & 1 \\
\hline \multirow[t]{3}{*}{ OC4888 } & & 4 & 4 & 2 & 1 \\
\hline & Range & $1 \sim 8^{*}$ & $2 \sim 8$ & & $0.5 \sim 2$ \\
\hline & Median & 4 & 4 & 4 & 1 \\
\hline \multicolumn{6}{|l|}{ S. aureus } \\
\hline ATCC29213 & & 0.5 & 0.5 & 0.5 & 0.5 \\
\hline ATCC13709 & & 0.5 & 0.5 & 0.5 & 0.5 \\
\hline \multirow[t]{4}{*}{ OC3726 } & & 0.5 & 0.5 & 0.5 & 0.5 \\
\hline & & $\cdots$ & $-\cdot$ & $\cdots-$ & $\cdots$ \\
\hline & Range & 0.5 & 0.5 & 0.5 & 0.5 \\
\hline & Median & 0.5 & 0.5 & 0.5 & 0.5 \\
\hline
\end{tabular}


Table 1 Continued

\begin{tabular}{|c|c|c|c|c|c|}
\hline & & \multicolumn{4}{|c|}{$\mathrm{MIC}(\mu \mathrm{g} / \mathrm{ml})$} \\
\hline & & $1^{\mathrm{a}}$ & 2 & $1^{\mathrm{b}}$ & 3 \\
\hline \multicolumn{6}{|c|}{ S. hemolyticus } \\
\hline OC3882 & ermC-inducible & $>16$ & $>16$ & $>16$ & $>16$ \\
\hline \multirow[t]{3}{*}{ OC4545 } & ermC-constitutive & $>16$ & $>16$ & $>16$ & $>16$ \\
\hline & Range & $>16$ & $>16$ & $>16$ & $>16$ \\
\hline & Median & $>16$ & $>16$ & $>16$ & $>16$ \\
\hline
\end{tabular}

${ }^{a}$ Measured on same day as for $\mathbf{2},{ }^{b}$ Measured on same day as for $\mathbf{3},{ }^{*}$ Combined ranges for both days.

\section{3-Chloropropanal (6)}

A solution of acrolein $(11.2 \mathrm{~g}, \quad 0.20 \mathrm{~mol})$ and dicinnamylacetone $(5 \mathrm{mg})$ in $100 \mathrm{ml}$ of $\mathrm{CH}_{2} \mathrm{Cl}_{2}$ was stirred and cooled on ice, and anhydrous $\mathrm{HCl}$ was bubbled into the solution until the color changed to red. The resulting solution was dried by addition of $\mathrm{MgSO}_{4}$, then filtered to provide a solution of 6 containing $<5 \%$ remaining acrolein. ${ }^{1} \mathrm{H}$ NMR $\left(400 \mathrm{MHz}, \mathrm{CDCl}_{3}\right) \delta 9.78(1 \mathrm{H}, \mathrm{t}, J=1.2 \mathrm{~Hz}$, $\mathrm{CHO}), 3.80\left(2 \mathrm{H}, \mathrm{t}, J=6.4 \mathrm{~Hz}, \mathrm{CH}_{2} \mathrm{Cl}\right), 2.93(2 \mathrm{H}, \mathrm{dt}, J=0.8$, $6.4 \mathrm{~Hz}, \mathrm{CH}_{2} \mathrm{CO}$ ).

The concentration was determined as described above for 4. This procedure provides an approximately $1.8 \mathrm{M}$ solution of 6 in $\mathrm{CH}_{2} \mathrm{Cl}_{2}$.

\section{( \pm )- $N$-[u-5-X-3-Hydroxy-2-methylpentanoyl]-2- benzoxazolones $(7,8,9)$}

Aldehydes $\mathbf{4} \sim \mathbf{6}$ were converted into their racemic syn $N$ - $[u$ 5-X-3-hydroxy-2-methylpentanoyl]-2-benzoxazolone aldol adducts 7 9, and the aldol adducts were converted into the thioesters 10 12 using the procedure described by [9]

\section{( \pm )- $N$-[u-5-Fluoro-3-hydroxy-2-methylpentanoyl]-2-} benzoxazolone (7)

White needles from methyl tert-butyl ether (MTBE), mp $117 \sim 118^{\circ} \mathrm{C} ;{ }^{1} \mathrm{H}$ NMR $\left(400 \mathrm{MHz}, \mathrm{CDCl}_{3}\right) \delta 8.07 \sim 8.02$ (m, $1 \mathrm{H}), 7.29 \sim 7.18(\mathrm{~m}, 3 \mathrm{H}), 4.77 \sim 4.05(\mathrm{~m}, 2 \mathrm{H}), 4.31(\mathrm{~m}$, $1 \mathrm{H}), 3.96(\mathrm{dq}, J=7,3 \mathrm{~Hz}, 1 \mathrm{H}), 2.00 \sim 1.79(\mathrm{~m}, 2 \mathrm{H}), 1.34$ $(\mathrm{d}, J=7 \mathrm{~Hz}, 3 \mathrm{H}) .{ }^{13} \mathrm{C}$ NMR $\left(100 \mathrm{MHz}, \mathrm{CDCl}_{3}\right) \delta 176.1$, $151.1,142.2,127.7,125.6,125.0,116.2,110.0,81.2$ $\left(\mathrm{d}, J_{\mathrm{CF}}=163 \mathrm{~Hz}\right), 68.0 \quad\left(\mathrm{~d}, J_{\mathrm{CF}}=4 \mathrm{~Hz}\right), 43.8,34.6(\mathrm{~d}$, $\left.J_{\mathrm{CF}}=19 \mathrm{~Hz}\right), 10.4$.

( \pm )- $N$-[u-5-Azido-3-hydroxy-2-methylpentanoyl]-2benzoxazolone (8)

White needles from 1:1 MTBE/hexane, mp $79 \sim 81{ }^{\circ} \mathrm{C} ;{ }^{1} \mathrm{H}$ NMR $\left(400 \mathrm{MHz}, \mathrm{CDCl}_{3}\right) \delta 8.08(1 \mathrm{H}, \mathrm{m}), 7.27(3 \mathrm{H}, \mathrm{m})$,
$4.23(1 \mathrm{H}$, ddd, $J=3.2,6.4,9.7 \mathrm{~Hz}), 3.96(1 \mathrm{H}, \mathrm{dq}, J=2.9$, $7.0 \mathrm{~Hz}), 3.52(2 \mathrm{H}, \mathrm{dd}, J=5.7,7.5 \mathrm{~Hz}), 2.79(1 \mathrm{H}, \mathrm{dd}, J=1.3$, $3.4 \mathrm{~Hz}), 1.83(1 \mathrm{H}, \mathrm{m}), 1.75(1 \mathrm{H}, \mathrm{m}), 1.34(3 \mathrm{H}, \mathrm{d}$, $J=6.8 \mathrm{~Hz}) .{ }^{13} \mathrm{C} \mathrm{NMR}\left(100 \mathrm{MHz}, \mathrm{CDCl}_{3}\right) \delta 176.0,151.1$, $142.2,127.6,125.6,125.0,116.3,110.0,68.8,48.4,43.8$, $32.9,10.5$.

\section{( \pm )- $N$-[u-5-Chloro-3-hydroxy-2-methylpentanoyl]-2- benzoxazolone (9)}

White needles from MTBE, mp $116 \sim 117^{\circ} \mathrm{C}$; ${ }^{1} \mathrm{H}$ NMR $\left(400 \mathrm{MHz}, \mathrm{CDCl}_{3}\right) \delta 8.07(1 \mathrm{H}, \mathrm{m}), 7.27(3 \mathrm{H}, \mathrm{m}), 4.36(1 \mathrm{H}$, ddd, $J=3.0,5.0,9.0 \mathrm{~Hz}), 3.97(1 \mathrm{H}, \mathrm{dq}, J=3.0,7.0 \mathrm{~Hz}), 3.74$ $(2 \mathrm{H}, \mathrm{m}), 2.83(1 \mathrm{H}, \mathrm{br} \mathrm{d}, J=3.0 \mathrm{~Hz}), 2.06(1 \mathrm{H}, \mathrm{m}), 1.89(1 \mathrm{H}$, m), $1.31(3 \mathrm{H}, \mathrm{d}, J=7.0 \mathrm{~Hz}) .{ }^{13} \mathrm{C}-\mathrm{NMR}\left(100 \mathrm{MHz}, \mathrm{CDCl}_{3}\right) \delta$ $176.1,151.1,142.2,127.6,125.6,125.0,116.2,110.0$, $68.4,43.7,41.7,36.4,10.6$.

\section{( \pm )- $u$-5-Fluoro-3-hydroxy-2-methylpentanoyl $N$ -}

\section{Propionylcysteamine Thioester (10)}

White amorphous solid, mp $42 \sim 43^{\circ} \mathrm{C} .{ }^{1} \mathrm{H}$ NMR $(400 \mathrm{MHz}$, $\left.\mathrm{CDCl}_{3}\right) \delta 5.96(1 \mathrm{H}, \mathrm{brs}), 4.64(1 \mathrm{H}, \mathrm{ddt}, J=47,9,5 \mathrm{~Hz})$, $4.58(1 \mathrm{H}, \mathrm{ddt}, J=47,9,5 \mathrm{~Hz}), 4.13(1 \mathrm{H}, \mathrm{dt}, J=9,4 \mathrm{~Hz})$, $3.50(2 \mathrm{H}, \mathrm{m}), 3.30(1 \mathrm{H}, \mathrm{br} \mathrm{s}), 3.04(2 \mathrm{H}, \mathrm{ddt}, J=19.4,14$, $6 \mathrm{~Hz}), 2.75(1 \mathrm{H}, \mathrm{dq}, J=4,7 \mathrm{~Hz}), 2.20(2 \mathrm{H}, \mathrm{q}, J=7 \mathrm{~Hz}), 1.8$ $(2 \mathrm{H}, \mathrm{m}), 1.23(3 \mathrm{H}, \mathrm{d}, J=7 \mathrm{~Hz}), 1.13(3 \mathrm{H}, \mathrm{t}, J=7 \mathrm{~Hz}) \cdot{ }^{13} \mathrm{C}$ NMR $\left(100 \mathrm{MHz}, \mathrm{CDCl}_{3}\right) \quad \delta$ 203.3, $174.9,81.5(\mathrm{~d}$, $\left.J_{\mathrm{CF}}=163 \mathrm{~Hz}\right), 68.7\left(\mathrm{~d}, J_{\mathrm{CF}}=4 \mathrm{~Hz}\right), 54.0,39.3,35.3(\mathrm{~d}$, $\left.J_{\mathrm{CF}}=19 \mathrm{~Hz}\right), 29.8,29.0,12.0,10.2$.

\section{( \pm )-u-5-Azido-3-hydroxy-2-methylpentanoate $N$ -} Acetylcysteamine Thioester (11)

Pale yellow oil. ${ }^{1} \mathrm{H}$ NMR $\left(400 \mathrm{MHz}, \mathrm{CDCl}_{3}\right) \delta 6.14(1 \mathrm{H}$, br s), $4.06(1 \mathrm{H}$, br d, $J=9.6 \mathrm{~Hz}), 3.40(4 \mathrm{H}, \mathrm{m}), 3.18(1 \mathrm{H}$, br s), $3.02(2 \mathrm{H}, \mathrm{m}), 2.72(1 \mathrm{H}, \mathrm{dq}, J=4.4,7.2 \mathrm{~Hz}), 1.98(3 \mathrm{H}$, s), $1.71(2 \mathrm{H}, \mathrm{m}), 1.24(3 \mathrm{H}, \mathrm{d}, J=6.8 \mathrm{~Hz}) .{ }^{13} \mathrm{C} \mathrm{NMR}$ $\left(100 \mathrm{MHz}, \mathrm{CDCl}_{3}\right) \delta 203.4,170.7,69.4,53.6,48.5,39.1$, 
$33.2,28.7,23.1,11.6$.

(士)-u-5-Chloro-3-hydroxy-2-methylpentanoyl $\mathrm{N}$ Acetylcysteamine Thioester (12)

Pale yellow oil. ${ }^{1} \mathrm{H}$ NMR $\left(400 \mathrm{MHz}, \mathrm{CDCl}_{3}\right) \delta 6.07(1 \mathrm{H}$, br s), $4.17(1 \mathrm{H}$, ddd, $J=3.0,6.4,10.0 \mathrm{~Hz}), 3.70(2 \mathrm{H}, \mathrm{m})$, $3.47(2 \mathrm{H}, \mathrm{m}), 3.08(3 \mathrm{H}, \mathrm{m}), 2.74(1 \mathrm{H}, \mathrm{dq}, J=4.0,7.2 \mathrm{~Hz})$, $1.98(3 \mathrm{H}, \mathrm{s}), 1.96(1 \mathrm{H}, \mathrm{m}), 1.80(1 \mathrm{H}, \mathrm{m}), 1.24(3 \mathrm{H}, \mathrm{d}$, $J=7.2 \mathrm{~Hz}) .{ }^{13} \mathrm{C} \mathrm{NMR}\left(100 \mathrm{MHz}, \mathrm{CDCl}_{3}\right) \delta 203.5,170.8$, $69.0,53.4,41.8,39.1,36.8,28.8,23.2,11.5$.

\section{Preparation of 15-X-6-Deoxyerythronolide B Analogs (13 15)}

A seed culture of Streptomyces coelicolor CH999/pJRJ2 was made by inoculating $1 \mathrm{ml}$ of frozen mycelium into a $250 \mathrm{ml}$ baffled flask containing $50 \mathrm{ml}$ of FKA medium (corn starch, $45 \mathrm{~g} /$ liter; corn steep liquor, $10 \mathrm{~g} /$ liter; dried, inactivated brewers yeast, $10 \mathrm{~g} /$ liter; and $\mathrm{CaCO}_{3}, 1 \mathrm{~g} /$ liter), $0.050 \mathrm{ml}$ of $50 \mathrm{mg} / \mathrm{ml}$ thiostrepton in DMSO (filter sterilized), and $0.500 \mathrm{ml}$ of $50 \%$ Antifoam B (JT Baker). The flask was incubated at $30^{\circ} \mathrm{C}$ with shaking at $175 \mathrm{rpm}$ for 48 hours (Innova floor shaker). The culture was transferred into a 2.8-liter baffled flask containing $500 \mathrm{ml}$ of FKA medium, $0.500 \mathrm{ml}$ of $50 \mathrm{mg} / \mathrm{ml}$ thiostrepton in DMSO, and $5 \mathrm{ml}$ of $50 \%$ Antifoam B, and the flask was incubated at $30^{\circ} \mathrm{C}$ with shaking at $175 \mathrm{rpm}$ for 48 hours.

A 10-liter stirred tank bioreactor (B. Braun) was autoclaved, filled with 5 liters of sterile FKA medium and $5 \mathrm{ml}$ of $50 \mathrm{mg} / \mathrm{ml}$ thiostrepton in DMSO, and then inoculated with $500 \mathrm{ml}(10 \%)$ of seed culture. The bioreactor was run for 24 hours at $30^{\circ} \mathrm{C}$ with stirring at $600 \mathrm{rpm}$, sparged with air at $1.33 \mathrm{LPM}$, and the $\mathrm{pH}$ was maintained at 6.50 by automated addition of $2.5 \mathrm{~N} \mathrm{NaOH}$ and $2.5 \mathrm{~N} \mathrm{H}_{2} \mathrm{SO}_{4}$.

Three liters of the above culture were used to inoculate a 100-liter bioreactor containing 55 liters of sterile FKA medium with $2 \mathrm{~g} /$ liter Tastone 310 added prior to sterilization. The initial fermentor agitation rate was set at a tip speed of $2.5 \mathrm{~m} / \mathrm{second}$, the temperature was maintained at $30^{\circ} \mathrm{C}$, the $\mathrm{pH}$ was controlled at $\mathrm{pH} 6.5$ by automated addition of $2.5 \mathrm{~N} \mathrm{NaOH}$ and $2.5 \mathrm{~N} \mathrm{H}_{2} \mathrm{SO}_{4}$, and the airflow was set at $0.4 \mathrm{vvm}$. Foaming was controlled by automated addition of $50 \%$ Antifoam B. During the fermentation, the dissolved oxygen was maintained at $>50 \%$ air saturation by cascade control using the agitation rate (tip speed of $2.5 \sim 3.0 \mathrm{~m} / \mathrm{second})$ and back pressure $(0.1 \sim 0.4$ bar $)$ in that order. After 24 hours post-inoculation, a $400 \mathrm{~g} /$ liter solution of the appropriate thioester $\mathbf{1 0} \sim \mathbf{1 2}$ in DMSO was added to a final concentration of $1 \mathrm{~g} /$ liter. Titers of $\mathbf{1 3} \sim \mathbf{1 5}$ were monitored by HPLC, and the culture was harvested by centrifugation when a maximum titer was reached.
The 13 15 was isolated by solid phase extraction. The broth was clarified by centrifugation and loaded onto a column containing HP-20 resin (Rohm and Haas) at a concentration of 1 liter resin/20 g 15-X-6-deoxyerythronolide B. The column was then equilibrated with 5 column volumes of water at a flow rate of $2 \sim 4 \mathrm{ml} / \mathrm{cm}^{2}$ minute. The loaded resin was washed with 2 column volumes of water followed by 2 column volumes of $30 \%$ methanol in water. The compound was eluted from the resin with methanol. The fractions containing desired compound were identified by HPLC with ELSD detection, then were pooled and the volatiles were removed under reduced pressure. The dried solids were extracted with $3 \sim 5$ liters of methanol and filtered to yield a solution containing $6 \sim 10 \mathrm{mg} / \mathrm{ml}$ of the compound, which was diluted with an equal volume of water. This solution was loaded onto a column of HP20SS (1 liter resin/20 g of compound), which was then washed with 2 column volumes of $50 \%$ aqueous methanol. The compound was then eluted with $70 \%$ methanol in water, and the fractions were analyzed by HPLC. Product-containing fractions were pooled and evaporated to dryness.

\section{5-Fluoro-6-deoxyerythronolide B (13)}

Crystals from ether/hexane, mp $122 \sim 123^{\circ} \mathrm{C} .[\alpha]_{\mathrm{D}}^{20}-62.2$ (c $0.4, \mathrm{CH}_{3} \mathrm{CN}$ ). IR $v_{\max }$ (thin film) $\mathrm{cm}^{-1} 1702,1176 .{ }^{1} \mathrm{H}$ NMR $\left(400 \mathrm{MHz}, \mathrm{CDCl}_{3}\right) \delta 5.49(1 \mathrm{H}, \mathrm{dd}, J=1.6,10.0 \mathrm{~Hz}$, H-13), 4.39 4.61 (2H, m; H-15), 3.99 (1H, m; H-5), 3.90 $(1 \mathrm{H}, \mathrm{d}, J=10.4 \mathrm{~Hz}, \mathrm{H}-3), 3.84(1 \mathrm{H}, \mathrm{d}, J=4.0 \mathrm{~Hz}, 11-\mathrm{OH})$, $3.70(1 \mathrm{H}$, ddd, $J=2.4,4.8,10.4 \mathrm{~Hz}, \mathrm{H}-11), 3.18(1 \mathrm{H}$, br s; 3-OH), 2.77 (2H, m; H-2+H-10), $2.61(1 \mathrm{H}, \mathrm{m} ; \mathrm{H}-8), 2.47$ (1H, br s; 5-OH), 2.10 2.25 (1H, m; H-14a), 2.00 (1H, m; H-6), 1.92 (1H, m; H-14b), 1.85 (m, 1H; H-4), $1.72(1 \mathrm{H}$, m; H-12), 1.67 (1H, ddd, $J=4.0,10.8,14.4 \mathrm{~Hz}, \mathrm{H}-7 \mathrm{a}), 1.29$ $(3 \mathrm{H}, \mathrm{d}, J=6.8 \mathrm{~Hz}, \mathrm{Me} 2), 1.26(1 \mathrm{H}, \mathrm{m} ; \mathrm{H}-7 \mathrm{~b}), 1.07(3 \mathrm{H}, \mathrm{d}$, $J=7.2 \mathrm{~Hz}, \mathrm{Me} 4), 1.06$ (3H, d, $J=6.4 \mathrm{~Hz}, \mathrm{Me} 8), 1.05$ (3H, d, $J=7.6 \mathrm{~Hz}, \mathrm{Me} 6), 1.02(3 \mathrm{H}, \mathrm{d}, J=6.8 \mathrm{~Hz}, \mathrm{Me} 10), 0.93(3 \mathrm{H}$, d, $J=6.8 \mathrm{~Hz}, \mathrm{Me} 12) .{ }^{13} \mathrm{C}$ NMR $\left(100 \mathrm{MHz}, \mathrm{CDCl}_{3}\right) \delta 213.7$ (C-9), 178.0 (C-1), 80.7 (d, $\left.J_{\mathrm{CF}}=166 \mathrm{~Hz}, \mathrm{C}-15\right), 79.3$ (C-3), 76.4 (C-5), 70.8 (d, $\left.J_{\mathrm{CF}}=4 \mathrm{~Hz}, \mathrm{C}-13\right), 70.7$ (C-11), 43.9 (C2), 43.3 (C-10), 41.1 (C-12), 39.5 (C-8), 37.6 (C-4), 37.5 (C-7), 35.5 (C-6), 33.3 (d, $\left.J_{\mathrm{CF}}=20 \mathrm{~Hz}, \mathrm{C}-14\right), 16.6$ (Me6), 14.6 (Me2), 13.3 (Me8), 9.2 (Me12), 6.9 (Me4), 6.3 (Me10). MS Found: $(\mathrm{M}+\mathrm{Na})^{+}$, 427.2456. $\mathrm{C}_{21} \mathrm{H}_{37} \mathrm{FO}_{6}$ requires $(\mathrm{M}+\mathrm{Na})^{+}, 427.2466$.

\section{5-Azido-6-deoxyerythronolide B (14)}

A mixture of $\mathbf{1 5}(19.07 \mathrm{~g}, \quad c a .88 \%$ pure $)$, sodium iodide $(5.95 \mathrm{~g})$, and sodium azide $(10.31 \mathrm{~g})$ in $60 \mathrm{ml}$ of dimethylsulfoxide was heated at $50^{\circ} \mathrm{C}$ for 3 days. The mixture was cooled to ambient temperature and diluted 
with ethyl acetate. The solution was washed with water, and the aqueous phase was extracted with ethyl acetate. The combined organic phases were washed with brine, dried over $\mathrm{MgSO}_{4}$, filtered, and evaporated. The residue was purified by silica gel chromatography using ethyl acetate/hexanes, yielding $13.96 \mathrm{~g}$ of 15-azido-6-dEB. Colorless needles from ether, mp $143 \sim 144^{\circ} \mathrm{C}$. $[\alpha]_{\mathrm{D}}^{20}-62.8$ (c 0.4, $\mathrm{CH}_{3} \mathrm{CN}$ ). IR $v_{\max }$ (thin film) $\mathrm{cm}^{-1} 2100,1710,1689$, 1178. ${ }^{1} \mathrm{H}$ NMR $\left(400 \mathrm{MHz}, \mathrm{CDCl}_{3}\right) \delta 5.39(1 \mathrm{H}, \mathrm{ddd}, J=1.4$, 2.9, $10.2 \mathrm{~Hz}, \mathrm{H}-13), 3.98(1 \mathrm{H}, \mathrm{m}, \mathrm{H}-5), 3.90(1 \mathrm{H}, \mathrm{dd}$, $J=2.8,10.4 \mathrm{~Hz}, \mathrm{H}-3), 3.80(1 \mathrm{H}, \mathrm{d}, J=4.0 \mathrm{~Hz}, 11-\mathrm{OH}), 3.68$ $(1 \mathrm{H}, \mathrm{ddd}, J=2.0,4.4,10.0 \mathrm{~Hz}, \mathrm{H}-11), 3.36(2 \mathrm{H}, \mathrm{m}, \mathrm{H}-15)$, $3.06(1 \mathrm{H}, \mathrm{d}, J=2.8 \mathrm{~Hz}, 3-\mathrm{OH}), 2.76(2 \mathrm{H}, \mathrm{m}, \mathrm{H}-2+\mathrm{H}-10)$, $2.61(1 \mathrm{H}, \mathrm{m}, \mathrm{H}-8), 2.29(1 \mathrm{H}, \mathrm{d}, J=3.6 \mathrm{~Hz}, \mathrm{OH}-5), 2.12$ $(1 \mathrm{H}, \mathrm{m}, \mathrm{H}-14 \mathrm{a}), 2.00(1 \mathrm{H}, \mathrm{m}, \mathrm{H}-6), 1.82(1 \mathrm{H}, \mathrm{dq}, J=1.6$, $5.6 \mathrm{~Hz}, \mathrm{H}-4), 1.70(2 \mathrm{H}, \mathrm{m}, \mathrm{H}-12+\mathrm{H}-14 \mathrm{~b}), 1.65(1 \mathrm{H}$, ddd, $J=3.6,10.4,14.0 \mathrm{~Hz}, \mathrm{H}-7 \mathrm{a}), 1.30$ (3H, d, $J=6.8 \mathrm{~Hz}, \mathrm{Me} 2)$, $1.26(1 \mathrm{H}$, ddd, $J=4.4,12.8,14.0 \mathrm{~Hz}, \mathrm{H}-7 \mathrm{~b}), 1.07(3 \mathrm{H}, \mathrm{d}$, $J=7.2 \mathrm{~Hz}, \mathrm{Me} 4), 1.06$ (3H, d, $J=6.0 \mathrm{~Hz}, \mathrm{Me} 8), 1.05(3 \mathrm{H}, \mathrm{d}$, $J=7.2 \mathrm{~Hz}, \mathrm{Me} 6), 1.02(3 \mathrm{H}, \mathrm{d}, J=6.8 \mathrm{~Hz}, \mathrm{Me} 10), 0.92(3 \mathrm{H}$, d, $J=6.8 \mathrm{~Hz}, \mathrm{Me} 12) .{ }^{13} \mathrm{C} \mathrm{NMR}\left(\mathrm{CDCl}_{3}, 100 \mathrm{MHz}\right): \delta 213.6$ (C-9), 178.0 (C-1), 79.3 (C-3), 76.4 (C-5), 71.8 (C-13), 70.7 (C-11), 48.5 (C-15), 43.9 (C-2), 43.2 (C-10), 41.2 (C12), 39.6 (C-8), 37.7 (C-4), 37.5 (C-7), 35.6 (C-6), 31.7 (C14), 16.6 (Me6), 14.7 (Me2), 13.4 (Me8), 9.3 (Me12), 6.9 (Me4), 6.3 (Me10). MS Found: $(\mathrm{M}+\mathrm{Na})^{+}, 450.2577$. $\mathrm{C}_{21} \mathrm{H}_{37} \mathrm{~N}_{3} \mathrm{O}_{6}$ requires $(\mathrm{M}+\mathrm{Na})^{+}, 450.2575$.

\section{5-Chloro-6-deoxyerythronolide B (15)}

Colorless needles from ether/hexane, mp $132 \sim 133^{\circ} \mathrm{C}$. IR $v_{\max }$ (thin film) $\mathrm{cm}^{-1} 1702,1173 .{ }^{1} \mathrm{H}$ NMR $(400 \mathrm{MHz}$, $\left.\mathrm{CDCl}_{3}\right) \delta 5.47(1 \mathrm{H}$, br d, $J=8.8 \mathrm{~Hz}, \mathrm{H}-13), 3.96(1 \mathrm{H}, \mathrm{m} ; \mathrm{H}-$ 5), $3.88(1 \mathrm{H}, \mathrm{dd}, J=2.8,10.4 \mathrm{~Hz}, \mathrm{H}-3), 3.84(1 \mathrm{H}, \mathrm{d}$, $J=4.4 \mathrm{~Hz}, 11-\mathrm{OH}), 3.67$ (1H, m; H-11), $3.53(2 \mathrm{H}, \mathrm{m}$; H15), $3.24(1 \mathrm{H}, \mathrm{d}, J=2.0 \mathrm{~Hz}, 3-\mathrm{OH}), 2.76(2 \mathrm{H}, \mathrm{m} ; \mathrm{H}-2+\mathrm{H}-$ 10), $2.75(1 \mathrm{H}, \mathrm{m} ; \mathrm{H}-8), 2.56(1 \mathrm{H}, \mathrm{br}$; $5-\mathrm{OH}), 2.30(1 \mathrm{H}, \mathrm{m}$; H-14a), 2.00 (1H, m; H-6), 1.88 (1H, m; H-14b), $1.70(1 \mathrm{H}$, $\mathrm{m} ; \mathrm{H}-12), 1.64(1 \mathrm{H}, \mathrm{m} ; \mathrm{H}-7 \mathrm{a}), 1.27(3 \mathrm{H}, \mathrm{d}, J=6.8 \mathrm{~Hz}$, Me2), $1.23(1 \mathrm{H}$, ddd, $J=4.0,13.6,13.6 \mathrm{~Hz}, \mathrm{H}-7 \mathrm{~b}), 1.04$ (3H, d, $J=7.2 \mathrm{~Hz}, \mathrm{Me} 4), 1.03$ (3H, d, $J=6.0 \mathrm{~Hz}, \mathrm{Me} 8), 1.02$ ( $3 \mathrm{H}, \mathrm{d}, J=7.2 \mathrm{~Hz}, \mathrm{Me} 6), 0.99$ (3H, d, $J=6.8 \mathrm{~Hz}, \mathrm{Me} 10$ ), $0.90(3 \mathrm{H}, \mathrm{d}, J=6.8 \mathrm{~Hz}, \mathrm{Me} 12) .{ }^{13} \mathrm{C}$ NMR $(100 \mathrm{MHz}$, $\left.\mathrm{CDCl}_{3}\right) \delta 213.7(\mathrm{C}-9), 178.0(\mathrm{C}-1), 79.3$ (C-3), 76.3 (C-5), 71.6 (C-13), 70.7 (C-11), 43.8 (C-2), 43.2 (C-10), 41.0 (C$12+\mathrm{C}-15), 39.5$ (C-8), 37.6 (C-7), 37.5 (C-4), 35.4 (C-6), 35.0 (C-14), 16.6 (Me6), 14.6 (Me2), 13.3 (Me8), 9.4 (Me12), 6.9 (Me4), 6.2 (Me10). MS Found: $(\mathrm{M}+\mathrm{Na})^{+}$, 443.2186. $\mathrm{C}_{21} \mathrm{H}_{37} \mathrm{ClO}_{6}$ requires $(\mathrm{M}+\mathrm{Na})^{+}$, 443.2171.

\section{Preparation of Erythromycin A analogs $(2,3)$}

A seed culture of Saccharopolyspora erythraea K39-14V was made by inoculating a $1 \mathrm{ml}$ aliquot of frozen mycelium into each of three $250 \mathrm{ml}$ baffled flasks containing $50 \mathrm{ml}$ of V1 medium (corn starch, $16 \mathrm{~g} /$ liter; corn dextrin, $10 \mathrm{~g} /$ liter; soya meal flour, $15 \mathrm{~g} /$ liter; corn steep liquor, $5 \mathrm{~g} /$ liter; soy bean oil, $6 \mathrm{~g} /$ liter; sodium chloride, $2.5 \mathrm{~g} /$ liter; ammonium sulfate, $1 \mathrm{~g} /$ liter; and $\mathrm{CaCO}_{3}, 4 \mathrm{~g} /$ liter) and $0.100 \mathrm{ml}$ of Antifoam B. The flasks were incubated at $34^{\circ} \mathrm{C}$ with shaking at $175 \mathrm{rpm}$ for 48 hours (Innova floor shaker). Each culture was transferred into a 2.8-liter baffled flask containing $500 \mathrm{ml}$ of $\mathrm{V} 1$ medium and $1 \mathrm{ml}$ of Antifoam B, and the flasks were incubated at $34^{\circ} \mathrm{C}$ with shaking at $175 \mathrm{rpm}$ for 48 hours.

Three 10-liter stirred tank bioreactors (B. Braun) were autoclaved, and each filled with 10 liters of sterile $50 \% \mathrm{~F} 1$ medium (corn starch, 17.5 g/liter; corn dextrin (type 3), $16 \mathrm{~g} /$ liter; soy meal flour, $16.5 \mathrm{~g} /$ liter; calcium carbonate, $4 \mathrm{~g} /$ liter, corn steep liquor, $6 \mathrm{~g} /$ liter; soy bean oil, $3 \mathrm{~g} /$ liter; sodium chloride $2.5 \mathrm{~g} / \mathrm{liter}$; and ammonium sulfate, $1 \mathrm{~g} /$ liter). The fermentor agitation rate was set at a tip speed of $2 \sim 4 \mathrm{~m} / \mathrm{second}$, the $\mathrm{pH}$ was controlled at $\mathrm{pH} 7.0$ by automated addition of $2.5 \mathrm{~N} \mathrm{NaOH}$ and $2.5 \mathrm{~N} \mathrm{H}_{2} \mathrm{SO}_{4}$, the temperature was maintained at $34^{\circ} \mathrm{C}$, and the airflow was set at $0.15 \mathrm{vvm}$. Foaming was controlled by automated addition of $50 \%$ Antifoam B. Each fermentor was inoculated with a 500-ml seed culture prepared above. During the fermentation, the dissolved oxygen was maintained at $>80 \%$ air saturation by cascade control using agitation rate (tip speed of $2 \sim 4 \mathrm{~m} /$ second), airflow $(0.15 \sim 0.5 \mathrm{vvm})$, and oxygen enrichment in that order. After 24 hours post-inoculation, a continuous $2 \mathrm{~g} /$ liter/day dextrin feed $(150 \mathrm{~g} /$ liter dextrin in deionized water) was initiated, and a $4 \% \mathrm{w} / \mathrm{v}$ solution of $\mathbf{1 3}$ or $\mathbf{1 4}$ was added to a final concentration of $250 \sim 300 \mathrm{mg} /$ liter. Samples were analyzed by HPLC every 12 hours. After cessation of erythromycin analog production ( $c a .60$ hours), the culture was harvested by centrifugation.

The erythromycin analog was isolated by solid phase extraction. The broth was adjusted to $\mathrm{pH} 9$ using $2.5 \mathrm{~N}$ $\mathrm{NaOH}$, clarified by centrifugation, and loaded onto a column containing HP-20 resin (Rohm and Haas) at a concentration of 1 liter resin $/ 20 \mathrm{~g}$ of erythromycin A analog. The column was then equilibrated with 5 column volumes of water at a flow rate of $2 \sim 4 \mathrm{ml} / \mathrm{cm}^{2}$-minute. The loaded resin was washed with 2 column volumes of water. The erythromycin analog was eluted from the resin with 5 column volumes of methanol. The fractions containing erythromycin analog were identified by HPLC, pooled, and the volatiles were removed under reduced pressure. The dried solids were mixed with $800 \mathrm{ml}$ of acetone and 3.2liter of hexane for 20 minutes. The mixture was then filtered using a \#4 Whatman filter paper. The solids were 
extracted twice in this manner, and the filtrates were combined and evaporated.

The crude product was dissolved in methanol and diluted with an equal volume of water. This solution was loaded onto a column of HP20SS (1 liter resin/20 g of erythromycin analog), which was then washed successively with 1 column volume of $50 \%$ aqueous methanol, 3 column volumes of $3: 2$ methanol/water, 3 column volumes of $7: 3 \mathrm{methanol} /$ water, 10 column volumes of $4: 1$ methanol/water, and finally 5 column volumes of $100 \%$ methanol. The fractions were analyzed by HPLC. Productcontaining fractions were pooled and evaporated to dryness.

\section{5-Fluoroerythromycin A (2)}

Crystals from $\mathrm{CH}_{3} \mathrm{CN}$ (solvate with 1.0 equivalent of $\left.\mathrm{CH}_{3} \mathrm{CN}\right)$, mp $175 \sim 176^{\circ} \mathrm{C} .[\alpha]_{\mathrm{D}}^{20}-77$ (c 1, EtOH). IR $v_{\max }$ (thin film) $\mathrm{cm}^{-1} 1734$ (s), 1720, 1699, 1165, 1052, 1012. ${ }^{1} \mathrm{H}$ NMR $\left(400 \mathrm{MHz}, \mathrm{CDCl}_{3}\right) \delta 5.29(1 \mathrm{H}, \mathrm{dd}, J=1.6$, $10.4 \mathrm{~Hz}, \mathrm{H}-13), 4.90\left(1 \mathrm{H}, \mathrm{d}, J=4.8 \mathrm{~Hz}, \mathrm{H}-1^{\prime \prime}\right), 4.49(2 \mathrm{H}, \mathrm{dt}$, $J=47.2,6.0 \mathrm{~Hz}, \mathrm{H}-15), 4.40\left(1 \mathrm{H}, \mathrm{d}, J=7.2 \mathrm{~Hz}, \mathrm{H}-1^{\prime}\right), 4.00$ $(1 \mathrm{H}, \mathrm{m}$; H-5"), $3.98(1 \mathrm{H}, \mathrm{d}, J=9.2 \mathrm{~Hz}, \mathrm{H}-3), 3.88(1 \mathrm{H}, \mathrm{s} ; \mathrm{H}-$ 11), $3.55(1 \mathrm{H}, \mathrm{d}, J=8.0 \mathrm{~Hz}, \mathrm{H}-5), 3.48\left(1 \mathrm{H}, \mathrm{m} ; \mathrm{H}-5^{\prime}\right), 3.32$ ( $3 \mathrm{H}, \mathrm{s}$; 3"-OMe), $3.23\left(1 \mathrm{H}, \mathrm{dd}, J=7.2,10.0 \mathrm{~Hz}, \mathrm{H}-2^{\prime}\right), 3.08$ $(1 \mathrm{H}, \mathrm{q}, J=7.2 \mathrm{~Hz}, \mathrm{H}-10), 3.00$ (1H, m; H-4"), $2.85(1 \mathrm{H}, \mathrm{dq}$, $J=7.2,9.2 \mathrm{~Hz}, \mathrm{H}-2), 2.70(1 \mathrm{H}, \mathrm{m} ; \mathrm{H}-8), 2.45(1 \mathrm{H}, \mathrm{m} ; \mathrm{H}-$ $\left.3^{\prime}\right), 2.36$ (1H, d, $\left.J=15.2 \mathrm{~Hz}, \mathrm{H}-2^{\prime \prime} \mathrm{a}\right), 2.32$ (1H, m; H-14a), $2.29\left(6 \mathrm{H}, \mathrm{s} ; \mathrm{NMe}_{2}\right), 2.05 \sim 1.80(3 \mathrm{H}, \mathrm{m} ; \mathrm{H}-4+\mathrm{H}-7 \mathrm{a}+\mathrm{H}-$ 14b), $1.73(1 \mathrm{H}, \mathrm{m} ; \mathrm{H}-7 \mathrm{~b}), 1.68\left(1 \mathrm{H}, \mathrm{m} ; \mathrm{H}^{-} 4^{\prime} \mathrm{a}\right), 1.58(1 \mathrm{H}$, dd, $\left.J=4.8,15.2 \mathrm{~Hz}, \mathrm{H}-2^{\prime \prime} \mathrm{b}\right), 1.47$ (3H, s; Me6), $1.31(3 \mathrm{H}, \mathrm{d}$, $\left.J=7.2 \mathrm{~Hz}, \mathrm{H}-6^{\prime \prime}\right), 1.24\left(3 \mathrm{H}, \mathrm{s} ; \mathrm{Me} 3^{\prime \prime}\right), 1.22(3 \mathrm{H}, \mathrm{d}$, $\left.J=6.0 \mathrm{~Hz}, \mathrm{H}-6^{\prime}\right), 1.16(6 \mathrm{H}, \mathrm{d}, J=6.8 \mathrm{~Hz}, \mathrm{Me} 2+\mathrm{Me} 8), 1.15$ (3H, s; Me12), 1.14 (3H, d, $J=6.4 \mathrm{~Hz}, \mathrm{Me} 10), 1.11$ (3H, d, $J=7.6 \mathrm{~Hz}, \mathrm{Me} 4) .{ }^{13} \mathrm{C} \mathrm{NMR}\left(100 \mathrm{MHz}, \mathrm{CDCl}_{3}\right) \delta 221.5(\mathrm{C}-$ 9), 175.5 (C-1), 103.2 (C-1'), 96.3 (C-1"), 83.4 (C-5), 83.2 (d, $\left.J_{\mathrm{CF}}=170 \mathrm{~Hz}, \mathrm{C}-15\right), 79.8$ (C-3), 77.9 (C-4"), 75.1 (C-6), $74.3(\mathrm{C}-12), 72.6\left(\mathrm{C}-3^{\prime \prime}\right), 72.5$ (d, $\left.J_{\mathrm{CF}}=4 \mathrm{~Hz}, \mathrm{C}-13\right), 70.9$ (C-2'), 68.9 (C-5'), 68.5 (C-11), 65.6 (C-3'+C-5"), 49.5 (3"-OMe), 45.2 (C-8), 44.8 (C-2), $40.3\left(\mathrm{NMe}_{2}\right), 39.6$ (C-4), 38.5 (C-7), 37.7 (C-10), $34.9\left(\mathrm{C}-2^{\prime \prime}\right), 29.4$ (d, $\left.J_{\mathrm{CF}}=20 \mathrm{~Hz}\right)$, 28.7 (C-4'), 27.0 (Me6), 21.5 (Me3"), 21.4 (C-6'), 18.6 (C6"), 18.2 (Me8), 16.2 (Me12), 15.3 (Me2), 11.9 (Me10), 9.1 (Me4). MS Found: $(\mathrm{M}+\mathrm{H})^{+}$, 752.4564. $\mathrm{C}_{37} \mathrm{H}_{66} \mathrm{FNO}_{13}$ requires $(\mathrm{M}+\mathrm{H})^{+}, 752.4591$.

\section{5-Azidoerythromycin A (3)}

Crystals from $\mathrm{CH}_{2} \mathrm{Cl}_{2}$ /hexanes, mp $135 \sim 137^{\circ} \mathrm{C}$. $[\alpha]_{\mathrm{D}}^{20}-78$ (c 1, EtOH). IR $v_{\max }$ (thin film) $\mathrm{cm}^{-1} 2100,1734,1722$, $1698,1164,1010 .{ }^{1} \mathrm{H}$ NMR $\left(400 \mathrm{MHz}, \mathrm{CDCl}_{3}\right) \delta 5.17(1 \mathrm{H}$, dd, $J=2.4,10.4 \mathrm{~Hz}, \mathrm{H}-13), 4.88\left(1 \mathrm{H}, \mathrm{d}, J=4.8 \mathrm{~Hz}, \mathrm{H}-1^{\prime \prime}\right)$, $4.40\left(1 \mathrm{H}, \mathrm{d}, J=7.2 \mathrm{~Hz}, \mathrm{H}-1^{\prime}\right), 4.00\left(2 \mathrm{H}, \mathrm{m} ; \mathrm{H}-3+\mathrm{H}-5^{\prime \prime}\right)$,
$3.84(1 \mathrm{H}, \mathrm{s} ; \mathrm{H}-11), 3.57(1 \mathrm{H}, \mathrm{d}, J=8.0 \mathrm{~Hz}, \mathrm{H}-5), 3.49(1 \mathrm{H}$, m; H-5'), 3.33 (3H, s; 3"-OMe), 3.33 (1H, m; H-15a), 3.21 (m, 2H; H-2'+H-15b), 3.08 (1H, q, $J=6.8 \mathrm{~Hz}, \mathrm{H}-10), 3.01$ (1H, dd, $\left.J=9.6,9.6 ; \mathrm{H}-4^{\prime \prime}\right), 2.86(1 \mathrm{H}, \mathrm{dq}, J=7.2,9.2 ; \mathrm{H}-2)$, $2.70(1 \mathrm{H}, \mathrm{m} ; \mathrm{H}-8), 2.42\left(1 \mathrm{H}, \mathrm{m} ; \mathrm{H}-3^{\prime}\right), 2.37(1 \mathrm{H}, \mathrm{d}$, $\left.J=15.2 \mathrm{~Hz}, \mathrm{H}-2^{\prime \prime} \mathrm{a}\right), 2.28\left(6 \mathrm{H}, \mathrm{s} ; \mathrm{NMe}_{2}\right), 2.20(1 \mathrm{H}, \mathrm{m} ; \mathrm{H}-$ 14a), 1.95 (2H, m; H-4+H-7a), $1.75(2 \mathrm{H}, \mathrm{m} ; \mathrm{H}-7 \mathrm{~b}+\mathrm{H}-$ 14b), $1.68\left(1 \mathrm{H}, \mathrm{m} ; \mathrm{H}-4^{\prime} \mathrm{a}\right), 1.56(1 \mathrm{H}, \mathrm{dd}, J=4.8,15.2 \mathrm{~Hz}$, H-2"b), 1.47 (3H, s; Me6), 1.28 (3H, d, J=7 Hz, H-6"), 1.24 $\left(3 \mathrm{H}, \mathrm{s} ; 3^{\prime \prime}-\mathrm{Me}\right), 1.22\left(3 \mathrm{H}, \mathrm{d}, J=7 \mathrm{~Hz}, \mathrm{H}-6^{\prime}\right), 1.19(3 \mathrm{H}, \mathrm{d}$, $J=7 \mathrm{~Hz}, \mathrm{Me} 2), 1.16(3 \mathrm{H}, \mathrm{d}, J=7 \mathrm{~Hz}, \mathrm{Me} 8), 1.13(3 \mathrm{H}, \mathrm{s}$; Me12), 1.12 (3H, d, $J=7 \mathrm{~Hz}, \mathrm{Me} 10), 1.11(3 \mathrm{H}, \mathrm{d}, J=7 \mathrm{~Hz}$, Me4). ${ }^{13} \mathrm{C}$ NMR (100 MHz, $\left.\mathrm{CDCl}_{3}\right) \delta 221.9$ (C-9), 175.4 (C-1), 103.2 (C-1'), 96.3 (C-1"), 83.3 (C-5), 79.8 (C-3), 78.0 (C-4"), 75.0 (C-6), 74.4 (C-12), 73.0 (C-13), 72.6 (C$\left.3^{\prime \prime}\right), 70.9\left(\mathrm{C}-2^{\prime}\right), 69.0\left(\mathrm{C}-5^{\prime}\right), 68.6(\mathrm{C}-11), 65.6\left(\mathrm{C}-5^{\prime \prime}\right), 65.6$ (C-3'), 49.5 (3"-OMe), 49.1 (C-15), 45.2 (C-8), 44.7 (C-2), 40.3 (2C, $\left.\mathrm{NMe}_{2}\right), 39.6$ (C-4), 38.5 (C-7), 37.7 (C-10), 34.9 (C-2"), 28.6 (C-4'), 27.8 (C-14), 26.9 (Me6), 21.5 (3"-Me), 21.4 (C-6'), 18.7 (C-6"), 18.2 (Me8), 16.2 (Me12), 15.6 (Me2), 12.0 (Me10), 9.1 (Me4). MS Found: $(\mathrm{M}+\mathrm{H})^{+}$, 775.4691. $\mathrm{C}_{37} \mathrm{H}_{66} \mathrm{~N}_{4} \mathrm{O}_{13}$ requires $(\mathrm{M}+\mathrm{H})^{+}$, 775.4699.

\section{Results and Discussion}

Diketide thioesters $\mathbf{1 0} \sim \mathbf{1 2}$ were prepared using a titaniummediated aldol condensation between $\mathrm{N}$-propionyl-2benzoxazolone and a 3-substituted propanal to give racemic syn-aldol adducts $\mathbf{7} \sim \mathbf{9}$. The 2-benzoxazolone auxiliary was readily displaced by the thiolate anion of either $N$ acetylcysteamine or $\mathrm{N}$-propionylcysteamine to provide the racemic syn diketide thioester (Scheme 1, R=Me or Et) [9]. The $\mathrm{N}$-propionylcysteamine thioester was chosen for $\mathbf{1 0}$ as this gave a more conveniently handled, solid material.

The required 3-azidopropanal (5) and 3-chloropropanal (6) were prepared by addition of the corresponding acids to acrolein, with the addition of $\mathrm{HCl}$ being titrated using dicinnamylacetone as indicator [10]. As an efficient synthesis and isolation of 3-fluoropropanal (4) had not been previously reported, a simple process suitable for the production of this unstable synthon in a form useable in the titanium-mediated aldol without purification was developed. Numerous conditions for oxidation of 3 -fluoropropanol were examined, yet most resulted in significant decomposition of the sensitive product or the generation of byproducts that were incompatible with the subsequent aldol reaction. TEMPO-catalyzed oxidation using $\mathrm{NaOCl}$ resulted in significant quantities of acrolein due to base-catalyzed elimination of HF, even under buffering with $\mathrm{NaHCO}_{3}$, and the large proportion of water 

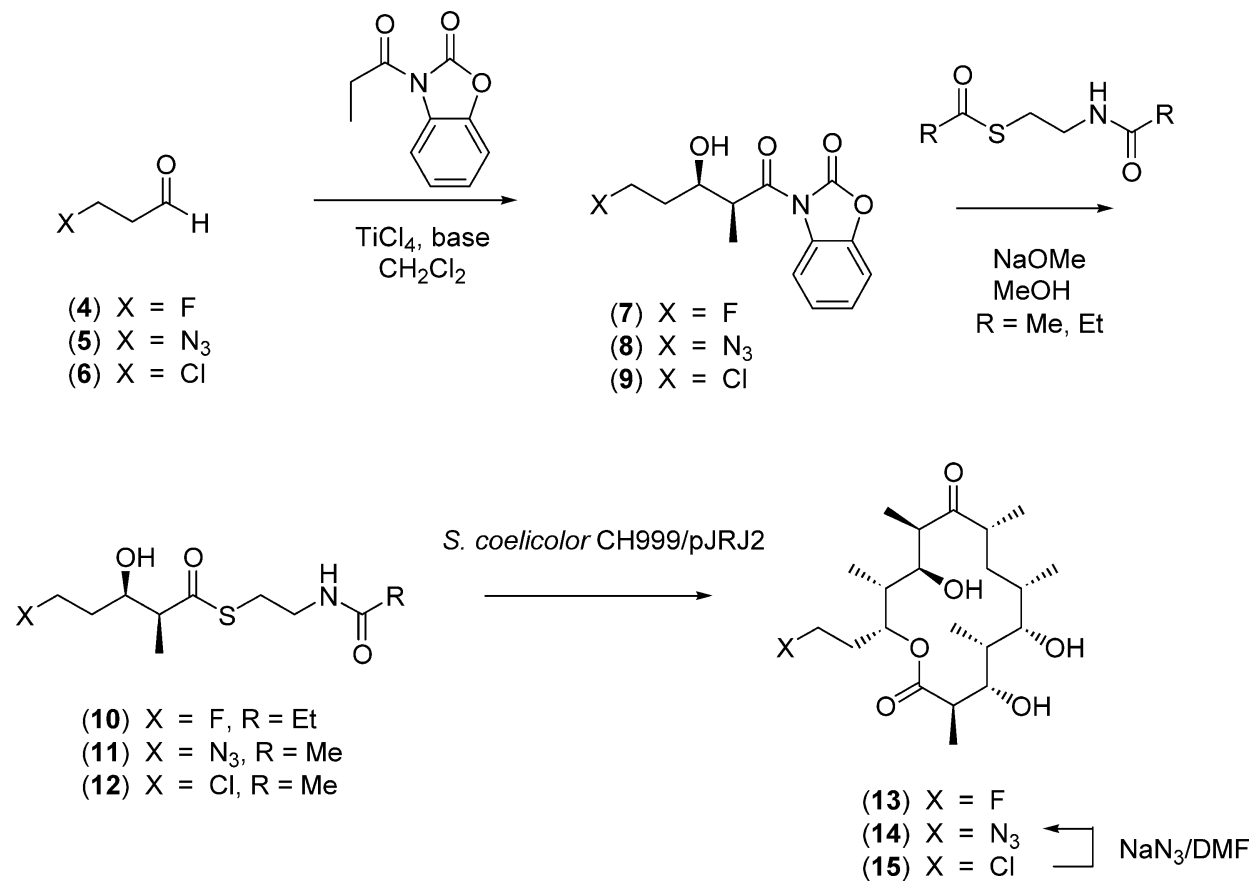

Scheme 1 Preparation of C-15 substituted 6-deoxyerythronolide B analogs.

involved led to low recoveries of the relatively watersoluble 4 . We found that the basicity of the reaction was ameliorated, and the proportion of water used lessened, by using trichloroisocyanuric acid as oxidant along with a slurry of $\mathrm{NaHCO}_{3}$ in a minimal volume of water in $\mathrm{CH}_{2} \mathrm{Cl}_{2}$. Thus, treatment of an equimolar mixture of 3fluoropropanol and $\mathrm{NaHCO}_{3}$ in $\mathrm{CH}_{2} \mathrm{Cl}_{2}$ containing $<10 \%$ $\mathrm{v} / \mathrm{v}$ water with catalytic TEMPO and one-third molar equivalent of trichloroisocyanuric acid, followed by decanting and drying of the resulting solution, provided a moderately stable solution of 4 in $\sim 80 \%$ yield.

The titanium-mediated aldol condensation between $\mathrm{N}$-propionyl-2-benzoxazolone and the base-sensitive aldehydes 4 or $\mathbf{6}$ gave improved results using $N$-methylmorpholine rather than triethylamine as base. Use of the weaker base minimized formation of the acrolein adduct. Azidopropanal 5 was efficiently condensed using triethylamine as base.

The racemic diketide thioesters $\mathbf{1 0} \sim \mathbf{1 2}$ were converted into the corresponding 6-deoxyerythronolide B analogs 13 $\sim \mathbf{1 5}$ using Streptomyces coelicolor CH999/pJRJ2 as previously described for other analogs [11]. While 14 was successfully prepared by feeding of $\mathbf{1 1}$, concerns over the large-scale synthesis of $\mathbf{5}$ led us to adopt an indirect route to this analog. Feeding of the chloride $\mathbf{1 2}$ produced $\mathbf{1 5}$ in good yield, demonstrating that the chemobiosynthesis process can tolerate a reactive primary alkyl halide. Conversion of $\mathbf{1 5}$ into azide $\mathbf{1 4}$ was readily accomplished by reaction with $\mathrm{NaN}_{3}$ in DMF.

Subsequent bioconversion of $\mathbf{1 3}$ and $\mathbf{1 4}$ using Saccharopolyspora erythraea K39-14V, an engineered mutant strain having an inactivated polyketide synthase, provided the erythromycin A analogs 2 and 3 [12]. The presence of the functional groups at the 15-position did not adversely affect the ability of the tailoring enzymes to act on these substrates, such that $\mathbf{1 3}$ and $\mathbf{1 4}$ were predominantly bioconverted into their erythromycin A analogs. Bioconversion of $\mathbf{1 5}$ provided only low titers of the corresponding 15-chloroerythromycin A.

The erythromycin A analogs described here show in vitro antibacterial activity comparable to that of erythromycin A as shown in Table 1. (As compounds $\mathbf{2}$ and $\mathbf{3}$ were tested on separate days, Table 1 gives a comparison of the MIC values with those for $\mathbf{1}$ measured on the same day as either 2 or 3.) The introduction of chemical handles at the 15position is itself not deleterious to antibacterial activity. Against macrolide-susceptible pneumococci, the MIC values of $\mathbf{2}$ and $\mathbf{3}$ were similar to those of $\mathbf{1}$. Activities against $m e f(A)$-containing pneumococci were comparable for $\mathbf{1}$ and $\mathbf{3}$, while MIC values for $\mathbf{2}$ were generally two-fold higher. With the exception of one pneumococcal strain, all compounds were relatively inactive against $\operatorname{erm}(B)$ containing $S$. pneumoniae or $S$. haemolyticus isolates, with MIC values $>16 \mu \mathrm{g} / \mathrm{ml}$. In contrast, 3 was selectively more active than the other two antibiotics against the Gramnegative respiratory pathogen $H$. influenzae, with MIC 


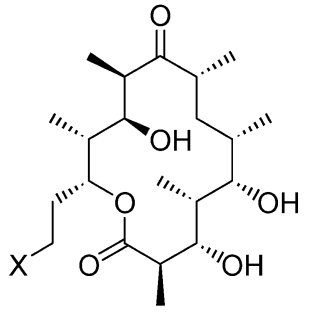

(13) $X=F$

(14) $X=\mathrm{N}_{3}$

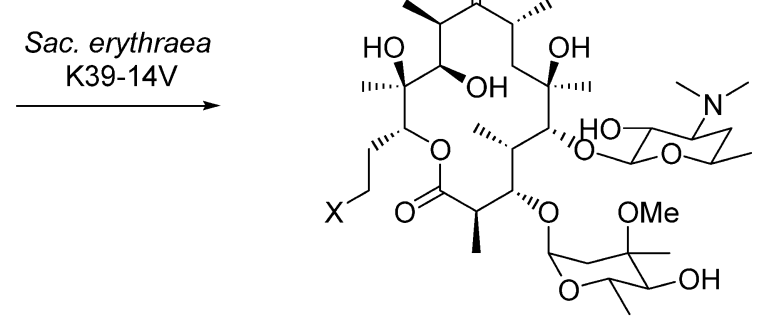

(2) $X=F$

(3) $X=N_{3}$

Scheme 2 Preparation of 15-fluoroerythromycin A and 15-azidoerythromycin A.

values two- to four-fold lower than $\mathbf{1}$ or $\mathbf{2}$.

Fluorine substitution is commonly used in medicinal chemistry to alter the lipophilicity or metabolic stability of a drug, and its inclusion often has beneficial effects on activity, protein binding, and cell penetration [13]. Flurithromycin, (8S)-8-fluoroerythromycin A, had higher serum and tissue levels than erythromycin at similar doses, demonstrative of the beneficial pharmacological effect of fluorination on the macrolide molecule [14]. Measurement of partitioning between 1-octanol and water at $\mathrm{pH} 7.4$ indicates that $2(\log \mathrm{D} \cong 0.3)$ is significantly less lipophilic than $\mathbf{1}(\log \mathrm{D} \cong 0.9)$. Thus, $\mathbf{2}$ is a scaffold for antibacterial development having unique pharmacological properties while retaining the fundamental bioactivity of $\mathbf{1}$.

Another erythromycin analog, $\mathbf{3}$, has a unique chemical handle that allows selective derivatization to create new analogs. Reduction of the azido group and subsequent functionalization of $\mathbf{3}$ has been recently described [15].

In summary, we have shown that chemobiosynthesis can provide novel analogs of erythromycin that are inaccessible by traditional chemical methods. These analogs contain unique functional groups that alter the physicochemical properties and allow for selective derivatization of the macrolide core, and so enable a new area of investigation into the generation of improved macrolide antibacterial agents.

\section{References}

1. Tanikawa T, Asaka T, Kashimura M, Misawa Y, Suzuki K, Sato M, Kameo K, Morimoto S, Nishida A. Synthesis and antibacterial activity of acylides (3- $O$-acyl-erythromycin derivatives): A novel class of macrolide antibiotics. J Med Chem 44: 4027-4030 (2001)

2. Jacobsen JR, Hutchinson CR, Cane DE, Khosla C. Precursor-directed biosynthesis of erythromycin analogs by an engineered polyketide synthase. Science 277: 367-369
(1997)

3. Frykman S, Leaf T, Carreras C, Licari P. Precursor-directed production of erythromycin analogs by Saccharopolyspora erythraea. Biotechnol Bioeng 76: 303-310 (2001)

4. Brown MS, Dirlam JP, McArthur HA, McCormick EL, Morse BK, Murphy PA, O'Connell TN, Pacey M, Rescek DM, Ruddock J, Wax RG. Production of 6-deoxy-13cyclopropyl-erythromycin B by Saccharopolyspora erythraea NRRL 18643. J Antibiot 52: 742-747 (1999)

5. McAlpine JB, Theriault RJ, Grebner KD, Hardy DJ, Fernandes PB. Minor products from the microbial transformation of 6-O-methylerythromycin A by Mucor circinelloides. Program and Abstracts of the 27th Intersci. Conf. on Antimicrob. Agents Chemother. No. 222, p. 134, New York, Oct. 4 7 (1987)

6. Adachi T, Sasaki J, Ōmura S. Hydroxylation and $N$ demethylation of clarithromycin (6-O-methylerythromycin A) by Mucor circinelloides. J Antibiot 42: 1433-1437 (1989)

7. National Committee for Clinical Laboratory Standards. Methods for dilution antimicrobial susceptibility tests for bacteria that grow aerobically, 5th Edition, Approved Standard M7-A5. National Committee for Clinical Laboratory Standards, Wayne, Pa. (2003)

8. Davies AJ, Donald ASR, Marks RE. The acid-catalysed decomposition of some $\beta$-azido-carbonyl compounds. J Chem Soc (C): 2109-2112 (1967)

9. Burlingame MA, Mendoza E, Ashley GW. N-Acyl-2benzoxazolinones in titanium-mediated aldol reactions. Tetrahedron Lett 45: 2961-2964 (2004)

10. Stowell JC, Keith DR, King BT. $\beta$-Halo acetals and -ketals: 2-(2-bromoethyl)-1,3-dioxane and 2,5,5-trimethyl-2-(2bromoethyl)-1,3-dioxane. Organic Syntheses 62: 140-148 (1984)

11. Leaf T, Burlingame M, Desai R, Regentin R, Woo E, Ashley G, Licari P. Employing racemic precursors in directed biosynthesis of 6-dEB analogs. J Chem Tech Biotech 77: 1122-1126 (2002)

12. Carreras C, Frykman S, Ou S, Cadapan L, Zavala S, Woo E, Leaf T, Carney J, Burlingame M, Patel S, Ashley G, Licari P. 
Saccharopolyspora erythraea-catalyzed bioconversion of 6deoxyerythronolide $\mathrm{B}$ analogs for production of novel erythromycins. J Biotechnol 92: 217-228 (2002)

13. Böhm H-J, Banner D, Bendels S, Kansy M, Kuhn B, Müller K, Obst-Sander U, Stahl M. Fluorine in medicinal chemistry. Chem Bio Chem 5: 637-643 (2004)

14. Benoni G, Cuzzolin L, Leone R, Consolo U, Ferronato G,
Bertrand C, Puchetti V, Fracasso ME. Pharmacokinetics and human tissue penetration of flurithromycin. Antimicrob Agents Chemother 32: 1875-1878 (1988)

15. Shaw SJ, Abbanat D, Ashley GW, Bush K, Foleno B, Macielag M, Zhang D, Myles DC. 15-Amido erythromycins: synthesis and in vitro activity of a new class of macrolide antibiotics. J Antibiot 58: 167-177 (2005) 